\title{
Mécanisme d'action et effets psychomimétiques de la phencyclidine (PCP) : relation avec les sites $P C P$ et sigma
}

La phencyclidine est un stupéfiant hallucinogène connu sous le nom de poussière d'ange. Il se lie à deux types de sites, PCP et sigma. Le premier est localisé dans le complexe récepteur/canal du NMDA, au niveau de la partie canalaire, et module négativement la réponse excitatrice au NMDA et au glutamate. Cet effet pourrait expliquer l'influence antagoniste de la phencyclidine et de ses dérivés sur la mémorisation, et son rôle protecteur contre les lésions neuronales post-anoxiques ou hypoglycémiques. La nature et le rôle des sites sigma sont moins bien connus. Dans certaines régions du cerveau, ils pourraient également interagir avec les récepteurs du NMDA. La phencyclidine active aussi le système dopaminergique par un mécanisme qui est encore largement indéterminé. Les mécanismes des effets psychomimétiques de cette drogue et de ses dérivés sont probablement complexes et restent à clarifier.

\section{Jean-Louis Junien Brian Leonard François J. Roman}

\author{
ADRESSES \\ J.-L. Junien : directeur de l'Institut de Recher- \\ che Jouveinal (IRJ). François J. Roman: \\ directeur de l'unité de biochimie pharmacologique \\ de l'IRJ. Institut de recherche Jouveinal, \\ 3-9, rue de la Loge, BP 100, 94265 Fres- \\ nes Cedex, France. \\ B. Leonard : professeur de psychopharmacologie, \\ University College Galway. Irlande du Nord. \\ Université de Galway, département de phar- \\ macologie, Irlande du Nord.
}

$\mathrm{m} / \mathrm{s} n^{\circ} 6$, vol. 7, juin-juillet 91

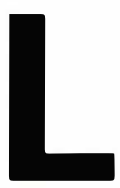

a phencyclidine (PCP) est une arylcyclohexylamine (phénylcyclohexylpipéridine), synthétisée pour la première fois en 1926 . Utilisée en clinique en 1958 comme anesthésique, son usage thérapeutique fut rapidement abandonné en raison des effets secondaires (hallucinations, excitation maniaque et pertes de mémoire) qu'elle entraînait chez les patients [1]. Depuis, la PCP poursuit sa carrière comme stupéfiant désigné par le terme de "poussière d'ange ". Ses propriétés ont très rapidement attiré l'attention des pharmacologues qui voyaient en ses effets une analogie avec certains symptômes de la schizophrénie. On a montré par la suite que des composés de la famille des benzomorphanes, qui se lient aux récepteurs des opiacés, telles la $\mathrm{N}$-allylnormétazocine (NANM) et la cyclazocine, entraînaient des effets analogues chez les animaux et l'homme. En pensant disposer d'un modèle expérimental de schizophrénie, les chercheurs se sont attachés à comprendre le mécanisme d'action de ces substances, et en particulier à essayer de caractériser des sites de liaison spécifiques pouvant rendre compte de leur(s) activité(s) pharmacologique(s) [2]. En raison du caractère opiacé des benzomorphanes, il a été initialement proposé d'utiliser le terme de sigma/opioïde pour définir ces sites. On devait montrer ultérieu- 


\section{RÉFÉRENCES}

1. Junien JL, Leonard B. Drugs acting on sigma and phencyclidine receptors : review of their nature, function and possible therapeutic importance. Clin Neuropharmacol 1989 ; 12 : 353-74.

2. Largent BL, Gundlach AL, Snyder SH. Pharmacological and autoradiographic discrimination of sigma and phencyclidine receptor binding sites in brain with $(+)-3 \mathrm{H}$ SKF 10,047 (+)-(3H)-3-(3-hydroxyphényl)$\mathrm{N}$-(1-propyl) piperidine and $(3 \mathrm{H})-1-(2-$ thienyl cyclohexyl) piperidine. I Pharmacol Exp Ther $1986 ; 238: 739-48$

3. Jarvis MF, Murphy DE, Williams M. Quantitative autoradiographic localization of NMDA receptors in rat brain using (3H)CPP : comparison with $(3 \mathrm{H}) \mathrm{TCP}$ binding sites. Eur $J$ Pharmacol $1987 ; 141$ : 149-52.

4. Collingridge GL, Singer W. Excitatory aminoacid receptors and synaptic plasticity. Trends Pharmacol Sci 1990 ; 11: 290-6.

5. Monaghan JB, Handemann GE, Flood WD, Cordi AA. D-cycloserine, a positive modulator of the N-methyl-Daspartate receptor enhances performance of the learning tasks in rats. Pharm Biochem Behav 1989 ; 34 : 649-53.

6. Gill R, Foster AC, Woodruff GB. Systemic administration of MK801 protects against ischemia induced hippocampal neurodegeneration in the gerbil. I Neurosci $1987 ; 7: 3343-9$

7. Tricklebank MD, Singh L, Oles RJ, Preston C, Iversen S. The behaviourial effects of MK801 : a comparison with antagonists acting non competitively and competitively at the .NMDA receptor. Eur $J$ Pharmacol $1989 ; 167$ : 127-35.

8. Balster RL. Substitution and antagonism in rats trained to discriminate $(+) \mathrm{N}$ allylnormetazocine from saline. J Pharmacol Exp Ther 1989 ; 3 : 749-56.

9. Wachtel H, Turski L. Glutamate : a new target in schizophrenia ? Trends Pharmacol Sci 1990 ; 11: 219-20.

10. Deutch AY, Tam SY, Freeman AS, Bowers MB, Roth RH. Mesolimbic and mesocortical dopamine activation induced by phencyclidine: contrasting pattern to striatal response. Eur $J$ Pharmacol 1987 ; 134: 257-64.

11. Rao TS, Kim HS, Lehman J, Martin LL, Wood PL. Selective activation of dopaminergic pathways in the mesocortex by coumpounds that act at the PCP binding site : tentative evidence for PCP recognition sites not coupled to N-methyl-Daspartate receptors. Neuropharmacology 1990 29 : 225-30.

12. French ED, Ceci A. Non competitive $\mathrm{N}$-methyl-D-aspartate antagonists are potent activators of ventral tegmental A10 dopamine neurons. Neurosci Lett 1990 ; 119 : 159-62.

13. Weber E, Sonders M, Quarum M, McLean S, Pou S, Keana JFW. 1,3-di(2-(5-3H)tolyl)guanidine : a selective ligand that labels sigma type receptors for psychotomimetic opiates and antipsychotic drugs. Proc Natl Acad Sci USA 1986 ; 83 : rement que la PCP et la NANM, ou plus spécifiquement son isomère dextrogyre la (+)NANM se liaient préférentiellement à deux sites différents dénommés respectivement sigma/PCP et sigma/opioïde. En réalité, le terme de récepteur sigma/opioïde était erroné, puisque les opiacés, à l'exception des benzomorphanes, ne présentent aucune affinité pour ce récepteur. On parle donc maintenant, d'une part, de sites sigma ou sigma/halopéridol, en raison de l'affinité particulière de l'halopéridol pour celui-ci, et, d'autre part, de sites récepteurs de la PCP, pour lesquels on dispose maintenant de ligands spécifiques.

\section{Sites PCP/NMDA}

Des études autoradiographiques à l'aide de ligands marqués tels que la [3H]TCP (thiénylcyclohexylpipéridine), un dérivé de la PCP, ont montré que la localisation des sites de haute affinité de la PCP se superpose à celle des récepteurs du NMDA (Nméthyl-D-aspartate) [2, 3], l'un des récepteurs du glutamate. Il existe une corrélation entre cette localisation et certains des effets fonctionnels des PCP mimétiques. En effet ces substances, dans les tests électrophysiologiques, biochimiques et pharmacologiques, se comportent comme des antagonistes vis-à-vis des effets du NMDA sans affecter ceux du quisqualate ou du kaïnate, deux autres acides aminés excitateurs.

La PCP et ses congénères se comportent comme des antagonistes non compétitifs, le site PCP étant différent du site NMDA lui-même (figure 1) et localisé dans le canal ionique activé par le NMDA. Ce canal est perméable au calcium et aux cations monovalents et il est normalement bloqué par les ions $\mathrm{Mg}^{2}+$ de façon dépendante du voltage. L'accession de la PCP à ses sites récepteurs est facilitée par l'ouverture de ces canaux lors de l'activation du site par le NMDA ou les substances favorisant l'activation du complexe telle la glycine $\left(\mathrm{m} / \mathrm{s} n^{\circ} 10\right.$, vol. 5, p. 781-2).

Les conséquences pharmacologiques et thérapeutiques éventuelles de cet effet des substances mimant l'action de la PCP sont liées au rôle physiologique du complexe récepteur/NMDA. On sait, par exemple, que le système NMDA est impliqué dans l'apprentissage et la mémoire. Il participe dans certaines régions de l'hippocampe au renforcement de l'efficience synaptique, phénomène appelé potentialisation à long terme (LTP) [4], qui servirait de support au stockage de l'information $(\mathrm{m} / \mathrm{s}$ $n^{\circ} 1$, vol. 4, p. 56). La PCP comme d'ailleurs les inhibiteurs compétitifs du NMDA, tels que le CPP [3-([ + ]-2-carboxypipérazine-4-yl)propyl-1-phosphonate] - exerce chez l'animal des effets amnésiants et bloque la potentialisation à long terme. Chez l'homme, la PCP provoque des pertes de mémoire. En revanche, les substances capables de renforcer l'activation du récepteur du NMDA comme la D-cyclosérine, qui se lie au site glycine, sont susceptibles d'améliorer les performances mnésiques chez l'animal [5].

A l'opposé de ces effets négatifs, les inhibiteurs de la transmission NMDAergique se comportent comme des neuroprotecteurs dans certaines conditions expérimentales. Ainsi, la dégénérescence neuronale observée au cours des anoxies cérébrales postischémiques, des hypoglycémies majeures, des privations d'oxygène au niveau de cultures de neurones, est liée à une libération excessive de glutamate et peut être limitée par des antagonistes du NMDA. Par exemple, la PCP et le MK801(dibenzocycloalkénimine), une substance se fixant également sur le site PCP mais de nature chimique différente, sont à même de s'opposer à la dégénérescence engendrée par ces traitements [6]. Les substances se fixant sur le site PCP/NMDA pourraient donc trouver leur place dans le traitement des accidents vasculaires où une baisse de l'irrigation cérébrale entraîne une destruction neuronale. Cette activité ne serait bien sûr utilisable que dans la mesure où leurs effets psychotiques seraient absents ou suffisamment dissociés.

\section{Sites PCP/NMDA et effets psychomimétiques}

L'idée qu'une neurotransmission glutaminergique altérée soit impliquée 
dans les phénomènes psychotiques, est argumentée par les effets comportementaux de la PCP, associés à son mécanisme d'action lié au NMDA. On peut rappeler aussi que le choc hypoglycémique à l'insuline était utilisé comme thérapeutique dans la schizophrénie avant l'arrivée des neuroleptiques; or il est bien connu maintenant que ce traitement produit une libération de glutamate au niveau cérébral. Chez le rongeur, les antagonistes du NMDA, qu'ils soient de nature compétitive ou non compétitive, produisent des anomalies identiques du comportement telles que l'hyperlocomotion, le retournement, le pédalage rétrograde et l'ataxie [7]. Les antagonistes compétitifs et non compétitifs se substituent au (+)NANM ou à la PCP chez des animaux entraînés à discriminer ces substances du sérum physiologique, indiquant qu'ils sont perçus par l'animal comme la PCP ou le ( +)NANM [8].

Pourtant, il n'existe chez l'homme aucune preuve que l'inhibition du complexe NMDA, que ce soit sur le site NMDA lui-même, le site de la glycine ou le site des polyamines, ait un effet psychomimétique. Il est donc prématuré de conclure à une relation directe entre inhibition du NMDA et effets psychotiques. De plus, la nature et la localisation de cette déficience restent hypothétiques. On pourra se reporter, à ce sujet, à l'article de Watchel et Turski [9].

\section{Sites PCP/NMDA et système dopaminergique}

L'activation du système dopaminergique dans la pathologie psychotique

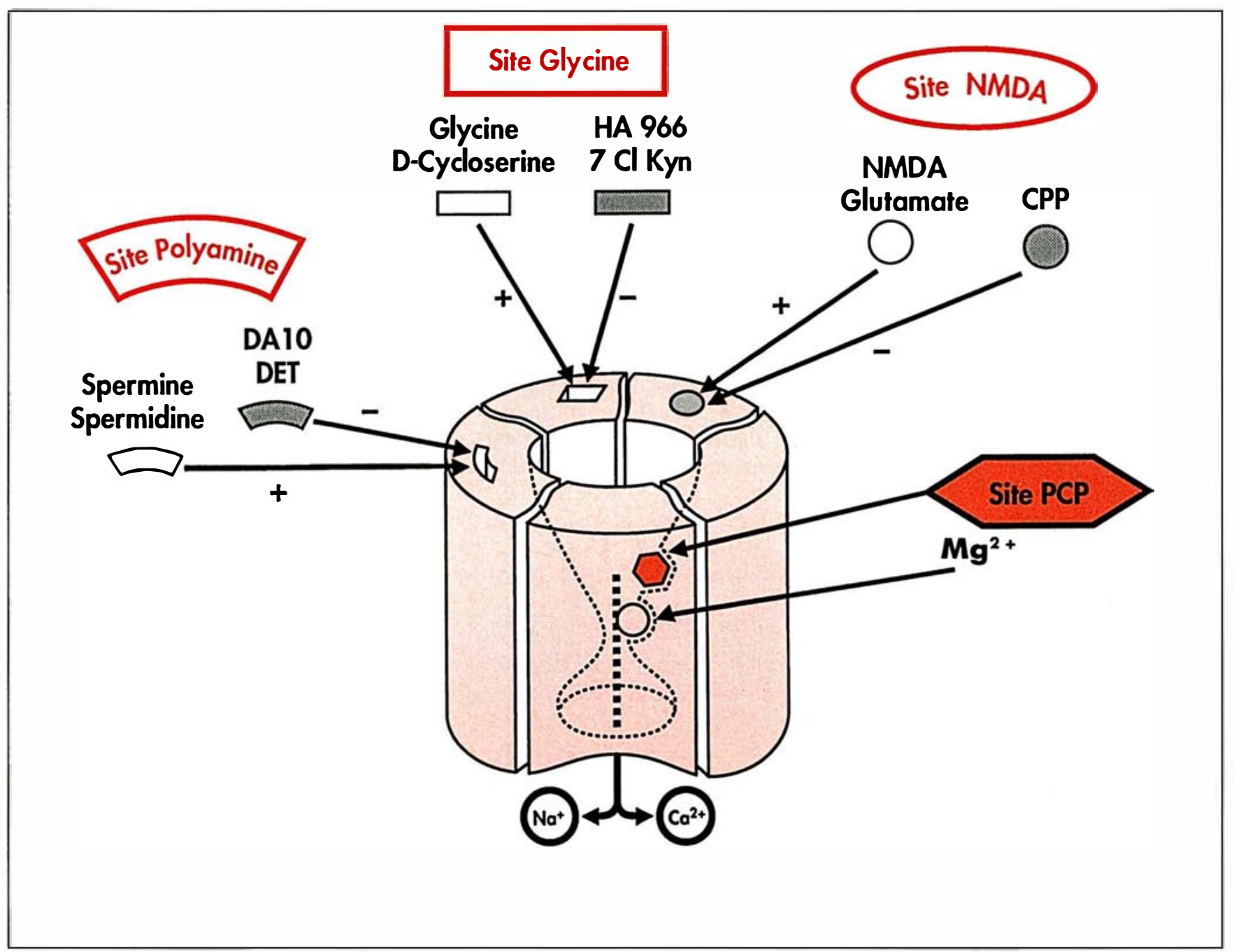

Figure 1. Récepteur au N-méthyl-D-aspartate (NMDA) et ses sites de régulation. Le site phencyclidine est localisé à l'intérieur du canal ionique. Le CPP [3-(1+)-2-carboxypipérazine-4-y/)-propyl-1-phosphonate] est un antagoniste du site NMDA. Le HA 966[3-amino-1-hydroxy-pyrrolidin-2-one] et la 7 chlorokyrurénine sont des antagonistes du site glycine. Le DA 10 11-10 diaminodécane) et le DET (diéthylène triamine) sont des antagonistes du site polyamine. La glycine, d'une part, et les polyamines, d'autre part, modulent positivement le complexe récepteur NMDA. 
14. Roman FJ, Pascaud X, Martin B, Vau ché $\mathrm{D}$, Junien JL. JO 1784, a potent and selective ligand for rat and mouse brain sigma sites. J Pharm Pharmacol 1990 ; 42 : 439-40. 15. Walker JM, Bowen WD, Walker FO Matsumoto RR, De Costa B, Rice KC. Sigma receptors : biology and function. Pharmacol Rev 1990 ; 42 : 4355-402.

16. Itzhak Y, Khouri M. Regulation of the binding of sigma and phencyclidine (PCP) receptor ligands in rat brain membranes by guanine nucleotides and ions. Neurosci Let $1988 ; 85$ : 147-52.

17. Matsumoto RR, Bowen WD, Walker JM. Down regulation of sigma receptors by chronic haloperidol. Prog Clin Biol Res 1989 ; 328 : 125-8.

18. Monnet FP, Debonnel G, Junien JL, de Montigny C. N-methyl-D-aspartate-induced neuronal activation is selectively modulated by sigma receptors. Eur J Pharmacol 1990 179 : 441-5.

19. Monnet FP, Debonnel G, de Montigny C. Neuropeptide Y selectively potentiates $\mathrm{N}$ methyl-D-aspartate-induced neuronal activation. Eur J Pharmacol 1990 ; 182 : 207-8.

20. Roman FJ, Pascaud X, Duffy O, Vauché D, Martin B, Junien JL. Neuropeptide Y and peptide $Y Y$ interact with rat brain sigma sites. Eur J Pharmacol 1989 ; 174 : 301-2. 21. Roman FJ, Pascaud X, Duffy O, Junien JL. N-methyl-D-aspartate receptor complex modulation by neuropeptide $\mathrm{Y}$ and peptide YY in rat hippocampus in vitro. Neurosci Let $1991 ; 122$ : 202-4.

22. Iyengard S, Mick S, Dilworth V, et al. Sigma receptors modulate the hypothalamic pituitary-adrenal (HPA) axis centrally : Evidence for a functional interaction with NMDA receptors, in vivo. Neuropharmacol $1990 ; 29: 299-303$.

23. Iyengar S, Dilworth VM, Mick SJ, et al. Sigma receptors modulate both $\mathrm{A} 9$ and A10 dopaminergic neurons in the rat brain : functional interaction with NMDA receptors. Brain Res 1990 ; 524 : 322-6

24. Contreras PC, Di Magio DA, O'Donohue TL, Lair CC. Biochemical and behavioral effects of sigma and PCP ligands. Synapse $1988 ; 2: 240-3$

25. Musacchio JM, Klein M, Canoll PD. Dextromethorphan and sigma ligands : Common sites but diverse effects. Life Sci 1989 ; $45: 1721-32$

26. Ferris RM, Tang FL, Chang KL, Russel A. Evidence that the potential antipsychotic agent rimcazole is a specific, competitive antagonist of sigma sites in brain. Life $\mathrm{Sc}$ $1986 ; 38: 2329-37$

27. Taylor DP, Dekleva J. Potential antipsychotic BMY 14802 selectively binds to sigma sites. Drug Dev Res 1987 ; 11 : 65-70.

28. Steinfels CF, Tam SW. Selective sigma receptor agonist and antagonist affect dopamine neuronal activity. EurJ Pharmacol 1989 ; $163: 167-70$

29. Beart PM, O'Shea RD, Manallack DT. Regulation of sigma receptors; high and low affinity agonist states, GTP shifts and up regulation by rimcazole and 1,3 di (2-tolyl)guanidine. J Neurochem $1989 ; 53$ : est largement documentée par l'efficacité thérapeutique des neuroleptiques antidopaminergiques. La PCP active les systèmes dopaminergiques mésolimbiques et mésocorticaux [10, 11] plus particulièrement impliqués dans les phénomènes psychotiques, mais affecte peu le système dopaminergique nigrostriatal [10]. La PCP stimule l'activité électrique des fibres dopaminergiques A10 qui se projettent de l'aire tegmentale ventrale dans le nucleus accumbens [12]. Ces effets sont partagés par les autres PCP mimétiques et montrent une bonne relation avec leur affinité pour le site PCP/NMDA. Cependant, Rao et al. [11] ont montré que, contrairement à ces substances, les antagonistes compétitifs du NMDA n'augmentent pas l'activité du système dopaminergique dans le mésocortex. Il n'a pas non plus été démontré que les antagonistes compétitifs activent le système dopaminergique mésolimbique. Ces résultats suggèrent que ces effets des PCP mimétiques ne sont pas dépendants d'un site PCP lié au NMDA, bien que celui-ci ait des caractéristiques de liaison voisines. Enfin, on sait depuis longtemps que la PCP inhibe la recapture de la dopamine [1]. Il est cependant peu probable que l'activation du système dopaminergique soit en relation avec cet effet puisque le MK801, qui se comporte comme la PCP, ne partage pas cette propriété. La nature du site impliqué reste donc à préciser.

\section{Sites sigma}

La PCP, comme nous l'avons mentionné précédemment, se fixe également sur les sites sigma préférentiellement marqués par le $(+)[3 \mathrm{H}]$ NANM [2]. L'hypothèse de l'intervention de ces sites dans les effets psychotiques de ces substances avait été confortée par la forte affinité de l'halopéridol et des butyrophénones neuroleptiques en général, ainsi que par leur présence dans les structures limbiques impliquées dans les réactions émotionnelles [2, 13]. La compréhension du rôle des sites sigma, qui reste encore partielle, a été longtemps freinée par l'absence de ligands spécifiques. Le ( +)NANM et la PCP présentent en effet l'inconvénient de se lier à la fois à ce site et au site PCP et l'halopéridol celui de se fixer aux récepteurs dopaminergiques, sérotoninergiques et adrénergiques. De nouveaux ligands plus sélectifs ont été proposés depuis, comme la di-ortho-tolyl guanidine (DTG) [13], le JO 1784 $[(+) \mathrm{N}$-cyclopropyl-mét hyl-Nméthyl-1, 4-diphényl-1-éthyl-but3-en-1-ylamine] [14] ou la 3(+)-PPP, $[(+)-3-(3-$ hydroxy phényl $)-\mathrm{N}$ (1-propyl)pipéridine] [2]. Ils ont permis de mieux étudier les caractéristiques et le rôle fonctionnel de ces sites. Il est aujourd'hui établi qu'il existe au moins deux sites sigma. Il a été montré en effet [15] que la liaison du $[3 \mathrm{H}](+)$ NANM, d'une part, et de la [3H]DTG, d'autre part, est modifiée de façon opposée par l'irradiation UV. La capacité relative des ligands à déplacer le $[3 \mathrm{H}](+)$ NANM ou le [3H]DTG varie. Walker et al. [15] ont ainsi proposé l'existence de deux sites soit indépendants, soit appartenant à un même complexe et interagissant de façon allostérique. La plupart des ligands - y compris l'halopéridol, la DTG ou le JO 1784 - se fixent avec une affinité plus ou moins grande sur ces deux sites, alors que le $(+)$ NANM n'a pratiquement pas d'affinité pour celui marqué par la [3H]DTG. Itzhak et Khouri [16] ont, par ailleurs, montré l'existence de deux composantes, de haute et basse affinité, dont la sensibilité aux guanines nucléotides est différente. La liaison de la DTG, du (+)NANM, de la (+)3-PPP est sensible aux dérivés du guanosine triphosphate (GTP), alors que celle de l'halopéridol ne l'est pas, comme on peut l'attendre d'un antagoniste. Il a été également montré qu'un traitement à l'halopéridol chez le rat pendant plusieurs jours diminuait de façon très sensible la liaison du $[3 \mathrm{H}](+)$ NANM ou d'un autre benzomorphane comme la $[3 \mathrm{H}](+)$ pentazocine. En revanche, ce traitement à l'halopéridol affecte peu la liaison de la [3H]DTG [17], ce qui, à nouveau, est en faveur de l'existence d'au moins deux sites sigma. Parmi les effets fonctionnels de ces composés, Monnet et al. [18] ont rapporté une potentialisation par la DTG, le JO 1784 et les benzomorphanes, des effets stimulants du NMDA sur l'activité électrique des 


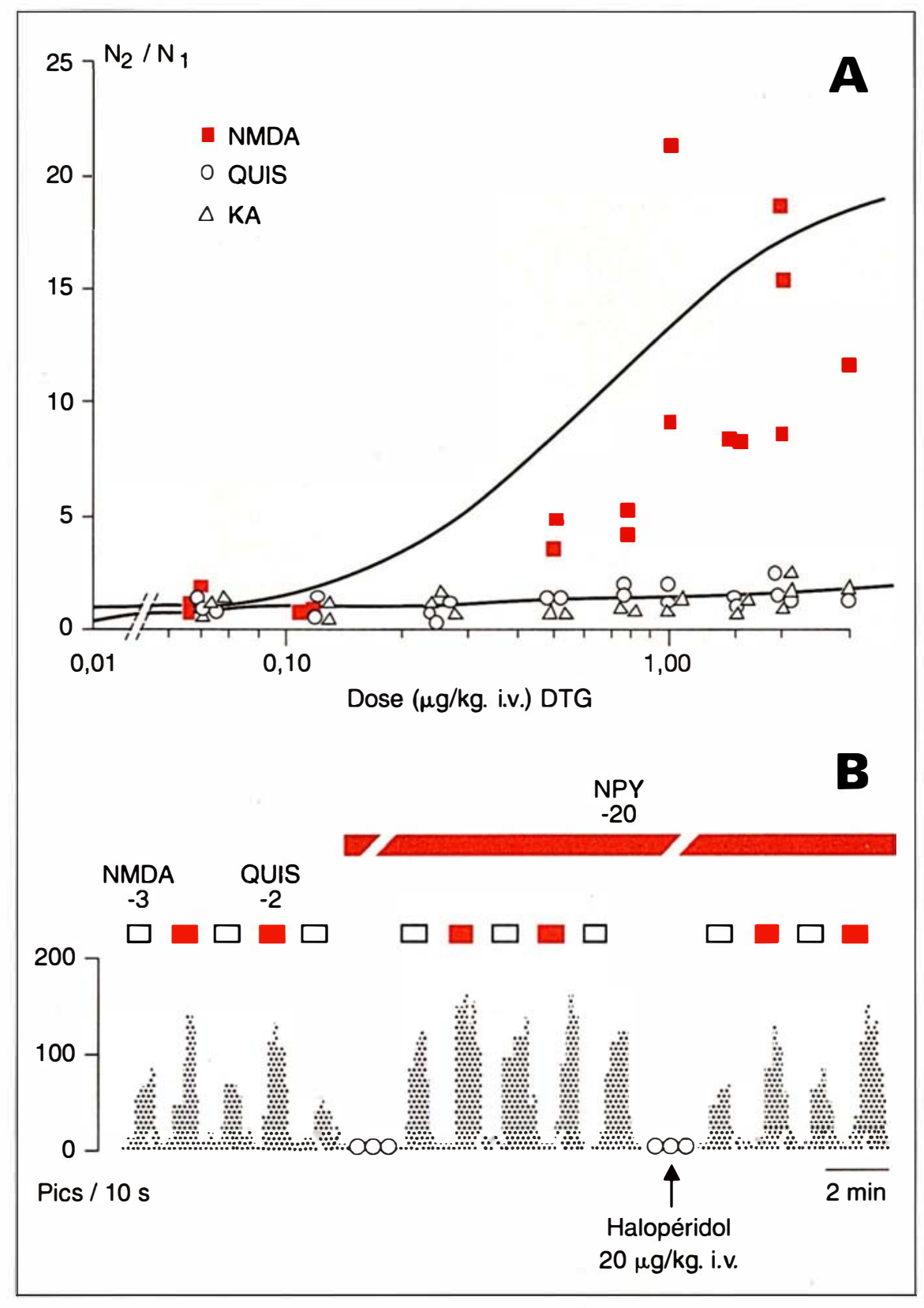

Figure 2. (A). Potentialisation de la réponse au NMDA par les ligands sigma dans la région CA3 de I'hippocampe. Le NMDA, le quisqualate (Quis) et le kainate $(\mathrm{Ka})$ sont appliqués par iontophorèse. N2/N1 représente le rapport entre le nombre de pics engendrés par $\mathrm{nC}$ (nanocoulon) par chacun des trois acides aminés, avant (N1) et 30 minutes après (N2) I'injection du ligand, ici la DTG. Chaque symbole représente l'effet d'une dose de DTG à un rat. On notera la sélectivité de l'effet sur le NMDA par rapport au Quis et au Ka. Cet effet est inhibé par l'halopéridol, mais pas par la spipérone, un antagoniste dopaminergique D2 qui n'a pas d'affinité pour le site sigma. (B). Effet du neuropeptide $Y$ (NPY) appliqué par micro-iontophorèse sur I'activation par le NMDA et le Quis des neurones pyramidaux de la région CA3 de I'hippocampe. Le NPY potentialise sélectivement la réponse au NMDA et cet effet est inhibé par l'halopéridol. La micro-iontophorèse permet l'injection de drogues ionisées pour tester leur action sur l'activité électrophysiologique du neurone. Les drogues sont mises en solution sous forme ionisée et retenues par un courant de rétention. L'inversion de la polarité du courant permet l'éjection de la drogue au niveau extracellulaire du neurone. -3 et -2 signifient nA (nanoampères).

$m / s n^{\circ} 6$, vol. 7 , juin-juillet 91 cellules pyramidales de la région $\mathrm{CA} 3$ de l'hippocampe chez le rat (figure 2). Cette potentialisation est retrouvée avec les peptides de la famille du neuropeptide Y (NPY) (un ligand endogène supposé) [19], qui ont également une forte affinité pour le site sigma marqué par le (+)NANM [20]. Une potentialisation induite par le NMDA de la libération de $[3 \mathrm{H}]$ noradrénaline à partir de coupes d'hippocampe de rat a été également décrite par Roman et al. [21] avec les ligands sigma, dont le NPY. Dans les deux cas, cette potentialisation est spécifiquement inhibée par l'halopéridol. La manière dont s'exerce l'interaction entre les sites de liaison sigma et le complexe récepteur NMDA reste inconnue. Selon certains auteurs [2, 13], les sites sigma ne font pas partie du complexe. En effet, le marquage autohistoradiographique des sites sigma, d'une part, et PCP/NMDA, d'autre part, n'est pas identique dans le cerveau.

Ce résultat suggère donc la possibilité, soit d'une interaction de type présynaptique, soit d'une interaction entre sites sur un même type cellulaire puisqu'il a été montré récemment [15] que les sites sigma comme les récepteurs NMDA sont largement présents sur les cellules pyramidales des régions CA3 et CA1 de l'hippocampe. Une relation avec le système NMDA a aussi été trouvée dans d'autres structures cérébrales. Ainsi, certains ligands sigma stimulent la sécrétion d'hormones hypophysaires [22] et cet effet est bloqué par l'administration de CPP, un antagoniste compétitif du NMDA. De la même manière, l'activation dopaminergique par les benzomorphanes dans le mésocortex [23] (voir plus loin) est inhibée par le CPP. Une meilleure connaissance de la nature et de la localisation des interactions sigmaNMDA est nécessaire, car la réponse à ces questions sous-tend le rôle des sites sigma via le récepteur du NMDA, et en conséquence leur rôle dans les processus mnésiques ou psychiques.

\section{Sites sigma et effets psychomimétiques}

Bien que Contreras et al. [24] aient rapporté l'apparition de stéréotypies 
et d'ataxie, après administration intracérébroventriculaire de DTG ou de PCP, ni la DTG ni la JO 1784 n'induisent, par voie systémique ou orale, les effets comportementaux des benzomorphanes et de la PCP. Ces composés, DTG et JO 1784, ne se substituent pas non plus au (+)NANM dans les expériences de discrimination chez le rongeur ([8] et communication personnelle). En fait, d'après Musachio et al. [25], ce sont les énantiomères $(-)$ des benzomorphanes, dont l'affinité sigma est moindre ou nulle, qui seraient responsables chez l'homme des effets observés avec les composés racémiques. Le dextrométhorphane, un morphinane qui est utilisé comme antitussif chez l'homme et qui a une forte affinité pour le site sigma, n'induit pas d'effet psychomimétique [25]. Certains produits en développement clinique ont revendiqué un mécanisme d'action antagoniste des récepteurs sigma tels le rimcazole [26] et le BMY 14802, une butyrophénone [27]. Cependant, le premier n'a qu'une affinité anecdotique pour ce site et le second possède une affinité forte pour les récepteurs 5 HT1A, ce qui limite sa spécificité pour le site sigma. Aucune de ces deux substances n'a pour le moment fait la preuve définitive de son activité antipsychotique. Ces données n'apportent donc pas d'arguments définitifs en faveur d'un rôle des sites sigma dans la pathologie psychotique.

\section{Sites sigma et système dopaminergique}

On retrouve les sites sigma dans les régions sensorimotrices où se distribue le système dopaminergique telles que les régions limbiques ou les ganglions basaux [15]. Plusieurs équipes ont cherché à mettre en évidence une relation fonctionnelle entre les deux systèmes. La DTG inhibe l'activité électrique du système dopaminergique nigrostriatal (A9) [28], mais ni la DTG ni le JO 1784 ne stimulent (ou n'inhibent) l'activité électrique des fibres dopaminergiques A 10 après administration aiguë par voie intraveineuse, contrairement à la $\mathrm{PCP}$ et au (+)NANM [12]. La DTG n'active pas non plus le métabolisme

tex [11] et dans le système mésolimbique [29], contrairement au (+)NANM, à la pentazocine [12, 28] et à la PCP [10-12]. D'après ces résultats, il semble donc que l'activation dopaminergique produite par ces composés ne soit pas un effet de leur affinité pour les sites sigma, mais plutôt un effet attribuable à leur affinité plus ou moins accusée pour le site PCP. Pourtant Iyengar et al. [23] ont récemment rapporté que l'augmentation du métabolisme de la dopamine, dans le striatum et les tubercules olfactifs (fibres A9 et A 10 dopaminergiques respectivement), produite par l'administration intracérébroventriculaire de $(+)$ NANM et de (+)pentazocine, était inhibée par la PCP. Ces auteurs concluent à une modulation par les récepteurs sigma des fibres dopaminergiques A9 et A10 via les récepteurs NMDA. Ce résultat s'écarte donc de celui obtenu avec les autres ligands sigma sélectifs qui, pourtant, sont également capables de moduler la réponse au NMDA dans l'hippocampe. Cette différence pourrait s'expliquer par la voie d'administration utilisée par Iyengar et al. [23] ou par le fait que ces ligands peuvent se fixer avec une affinité variable aux différents soustypes de sites sigma mentionnés précédemment et en moduler les activités respectives.

\section{Conclusion}

Les récents travaux sur la PCP et ses dérivés ont permis de mieux connaître le point d'impact de ces substances à l'échelon moléculaire. L'interaction de la PCP avec le récepteur du NMDA est clairement établie. Cependant, le rôle de cette interaction dans les effets psychotiques de la substance semble incertain. L'implication du site sigma, dont le rôle fonctionnel est moins connu, mais qui lui aussi contrôle l'activité du NMDA dans certaines régions du cerveau, reste également hypothétique. L'origine commune ou non de la puissante activation du système dopaminergique mésocortical et limbique produite par la PCP et les benzomorphanes reste cependant à clarifier, afin d'espérer la découverte de nouvelles drogues susceptibles de traiter les troubles psychotiques

\section{Summary}

Mechanism of action and psychomimetic effects of phencyclidine (PCP) : role of $\mathbf{P C P}$ and sigma sites

Phencyclidine (PCP, angel dust) is a major drug of abuse that produces many undesirable effects, some resembling schizophrenia. There has been extensive work in the last decade to characterize its receptor binding profile and to understand its mechanism of action. PCP binds to several sites including PCP and sigma sites originally suspected to mediate its psychomimetic effects. The PCP site is now known to belong to the $\mathrm{N}$-methyl-D-aspartate (NMDA) receptor complex and, as such, to modulate negatively the NMDA glutamate response. The role of the sigma site is less understood but recent reports have shown that sigma ligands also interfere with the NMDA response in some brain regions. In contrast to sigma ligands, the competitive NMDA antagonists and PCP produce similar behavioural effects in rodents. For example, the drugs substitute for each other in drug discrimination paradigms. However, neither the competitive NMDA antagonists nor the more specific sigma ligands stimulate the mesocortical, mesolimbic dopaminergic pathways as observed with PCP and benzomorphans. Although the functional role of $\mathrm{PCP} / \mathrm{NMDA}$ and sigma sites is now better understood, the origin of the psychomimetic effects and dopaminergic activation induced by PCP and benzomorphans remains to be clarified.

\section{TIRÉS A PART}

J.-L. Junien. 